Journal of Engineering and Applied Sciences 5 (1): 14-18, 2010

ISSN: 1816-949X

(C) Medwell Journals, 2010

\title{
Numerical Solving of Poisson Equation in 3D Using Finite Difference Method
}

\author{
${ }^{1}$ Sefer Avdiaj and ${ }^{2}$ Janez Setina \\ ${ }^{1}$ C-07-13, South City Condomenium, Serdang Perdana, Serikembengan 43300, \\ Serdang, Selngor, Darul Ehsan, Malaysia \\ ${ }^{2}$ Institute of Metals and Technology, Lepi pot 11, Ljubljana, Slovenia
}

\begin{abstract}
Scientists and engineers use several techniques in solving continuum or field problems. Loosely speaking, these techniques can be classified as experimental, analytical or numerical. Experiments are expensive, time consuming, sometimes hazardous and usually do not allow much flexibility in parameter variation. However, every numerical method, as we shall see, involves an analytic simplification to the point where it is easy to apply the numerical method. In spite of this fact, the following methods are among the most commonly used in Electro Magnetism (EM). In general these methods could be divided in: Analytical Methods and Numerical Methods. Application of these methods is not limited to EM-related problems; they find applications in other continuum problems such as in fluid, heat transfer and acoustics. In this study, the FDM has been elaborated. In the beginning approximate methods in general have been elaborated. The finite difference techniques are based upon approximations which permit replacing differential equations by finite difference equations. These finite difference approximations are algebraic in form; they relate the value of the dependent variable at a point in the solution region to the values at some neighboring points. From the results we can see that for case when we have uniform distribution of the electric charges inside the cube, we obtain maximum of the potential in the center of cube, whereas if we have inside the cube only one electric charge we will obtain so-called Green function. From the results we can see that the accuracy increases with increasing the number of grid points and iterations.
\end{abstract}

Key words: Finite difference method, poisson equation, Laplace's equation, electric charges, numerical methods, analytical methods

\section{INTRODUCTION}

In this study, we consider the solution of the Poisson equation on a regular $3 \mathrm{D}$ domain. An important application of finite differences is in numerical analysis especially in numerical differential equations which aim at the numerical solution of ordinary and partial differential equation, respectively. The idea is to replace the derivatives appearing in the differential equation by finite differences that approximate them. The resulting methods are called finite difference methods.

The main problem of electrostatics is solving Poisson equation:

$$
\Delta V=-\rho / \varepsilon_{0}
$$

This equation is a simply a combination of Gauss's law with the fact:

$$
\mathrm{E}=-\nabla \mathrm{V}
$$

In the regions where there are no charges, thus the Poisson's equation transforms into Laplace's equation:

$$
\Delta \mathrm{V}=0
$$

(Jackson, 1999). The most common situations are when the potential on the surface that surrounds the area of interest is known.

Using the Poisson's equation we can determine the potential within the area. To solve numerically these equations we should do approximations of partial derivative by differences. Using Finite Difference Method (FDM) we can solve also diffusion equation (especially Fick's second low) which is the motivation of this study because the Fick's second low is very useful in vacuum technology and our research is focused in this field.

\section{MATERIALS AND METHODS}

Finite difference method: Is one of the methods for solving Poisson's equation.Partial differential equations that describe the electrostatic field, under this method transformed algebraic equations systems which then

Corresponding Author: Sefer Avdiaj, C-07-13, South City Condomenium, Serdang Perdana, Serikembengan 43300, Serdang, Selngor, Darul Ehsan, Malaysia 
solved through software. The finite difference technique is based upon approximation which permits replacing

differential equations by finite difference equations.

These finite difference approximations are algebraic in form; they relate the value of the dependent variable at a point in the solution region to the values at some neighboring points. Thus a finite difference solution basically involves three steps (Sadiku, 2001):

- Dividing the solution region into a grid of nodes

- Approximation the given differential equation by finite difference equivalent that relates the dependent variable at a point in the solution region to its values

- solving the difference equations subject to the prescribed boundary conditions and/or initial conditions

The course of action taken in three steps is dictated by the nature of the problem being solved, the solution region and the boundary conditions (Roberts, 2001; Smedingho, 2005). The most commonly used grid patterns for two-dimensional problems are shown in Fig. 1.

Examine the point $\mathrm{M}(\mathrm{x}, \mathrm{y})$ on two dimensions electrostatic field and assume that the potential of this point is $\mathrm{V}(\mathrm{x}, \mathrm{y})$.

Potential of close points to M1, M2, M3 and M4 indicated in Fig. 2 can be determined approximately if the potential $\mathrm{V}(\mathrm{x}, \mathrm{y})$ transformed into Taylor series with respect the $\mathrm{x}$ and $\mathrm{y}$ axis, so that for practical reasons, the infinite series is truncated after a second-order term (Pang, 2006).

$$
\begin{aligned}
& V(x+a, y)=V(x, y)+a \frac{\partial V}{\partial x}+\frac{a^{2}}{2} \cdot \frac{\partial^{2} V}{\partial x^{2}}+\ldots \ldots \\
& V(x-a, y)=V(x, y)-a \frac{\partial V}{\partial x}+\frac{a^{2}}{2} \cdot \frac{\partial^{2} V}{\partial x^{2}}+\ldots \ldots
\end{aligned}
$$

After collect the last two equations side to side is obtained:

$$
\frac{\partial^{2} V}{\partial x^{2}}=\frac{V(x+a, y)+V(x-a, y)-2 \cdot V(x, y)}{a^{2}}
$$

Similarly will win even during the determine of the potential $\mathrm{V}(\mathrm{x}, \mathrm{y})$ with respect to $\mathrm{y}$ axis:

$$
\frac{\partial^{2} V}{\partial y^{2}}=\frac{V(x, y+a)+V(x, y-a)-2 \cdot V(x, y)}{a^{2}}
$$

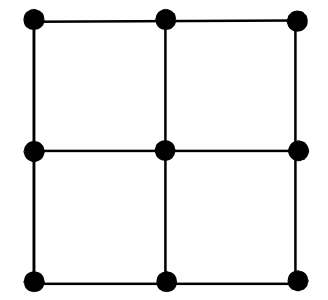

(a)

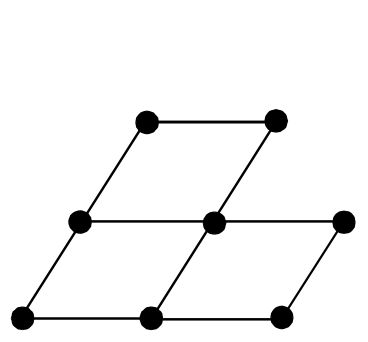

(c)

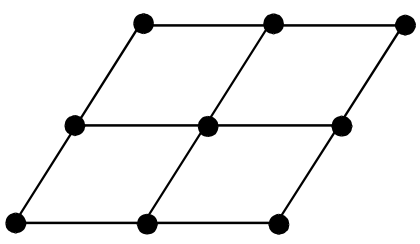

(b)

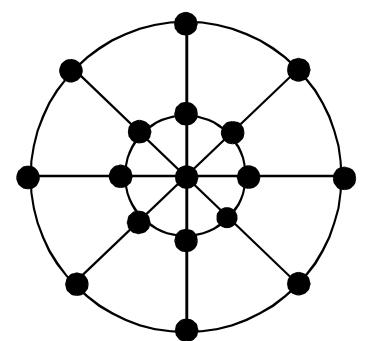

(d)
Fig. 1: Common grid patterns: (a). rectangular grid, (b) skew grid. (c) triangular grid. (d)circular grid

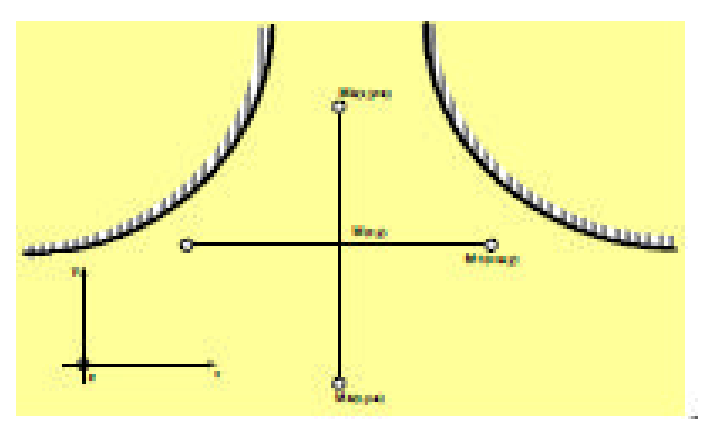

Fig. 2: Show potential of close points to $M_{1}, M_{2}, M_{3}$ and $\mathrm{M}_{4}$

Application of on FDM ne Poisson's Equation: Poisson equation is:

Where:

$$
\nabla^{2} \mathrm{~V}=-\rho / \varepsilon
$$

$\rho=$ Charge density

$\varepsilon=$ Permittivity of the medium

If the charge density is zero, then Laplace's equation results, the right side of the last equation becomes zero and we thus obtain:

$$
\nabla^{2} \mathrm{~V}=0
$$

The approximation of Laplace's equation by difference method is:

$$
\frac{\partial^{2} \mathrm{~V}}{\partial \mathrm{x}^{2}}+\frac{\partial^{2} \mathrm{~V}}{\partial \mathrm{y}^{2}}=0
$$




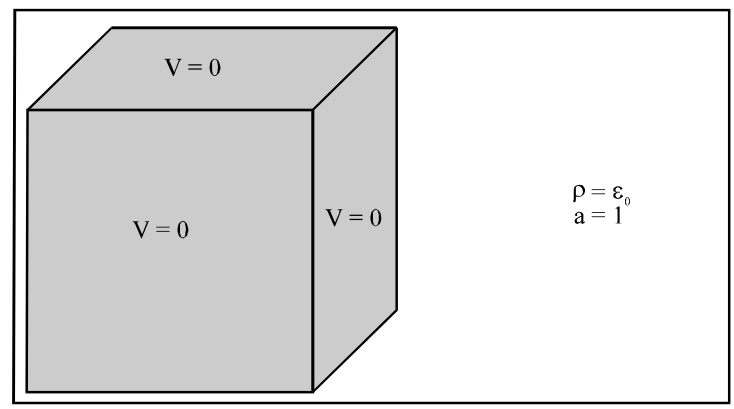

Fig. 3: Boundary condition

Substituting Eq. 3 and 4, into (7) gives:

$$
\begin{aligned}
& V(x, y)=\frac{1}{4} \cdot(V(x+a, y)+V(x-a, y)+ \\
& +V(x, y+a)+V(x, y-a))
\end{aligned}
$$

So this is the potential in the point $\mathrm{M}(\mathrm{x}, \mathrm{y})$.

By comparing with two-dimensional case, the Laplace's equation for the three-dimensional will be:

$$
\begin{aligned}
& V(x, y, z)=\frac{1}{6} \cdot(V(x+a, y, z)+V(x-a, y, z)+ \\
& +V(x, y+a, z)+V(x, y-a, z)+V(x, y, z+a) \\
& +V(x, y, z-a))
\end{aligned}
$$

The same way can be used to calculate the potential from Poisson's equation, using the same approximation for $\Delta \mathrm{V}$ and adding on every point electric charges density, so the difference approximation, the equation for potential on the point $\mathrm{M}(\mathrm{x}, \mathrm{y})$ becomes:

$$
\begin{aligned}
& V(x, y, z)=\frac{1}{6} \cdot(V(x+a, y, z)+V(x-a, y, z)+ \\
& +V(x, y+a, z)+V(x, y-a, z)+V(x, y, z+a) \\
& \left.+V(x, y, z-a)+\frac{a^{2}}{\varepsilon} \cdot \rho(x, y, z)\right)
\end{aligned}
$$

Boundary condition: We will assume that side of the cube they will be with zero potential whereas inside the cube has electrical charge distribution. It will also assume that the density of electric charges is $\rho=\varepsilon$ and the $a=1$. Fig. 3 .

\section{RESULTS AND DISCUSSION}

The results are given for two cases of boundary condition:

- Inside the cube the density charges are uniform distributed

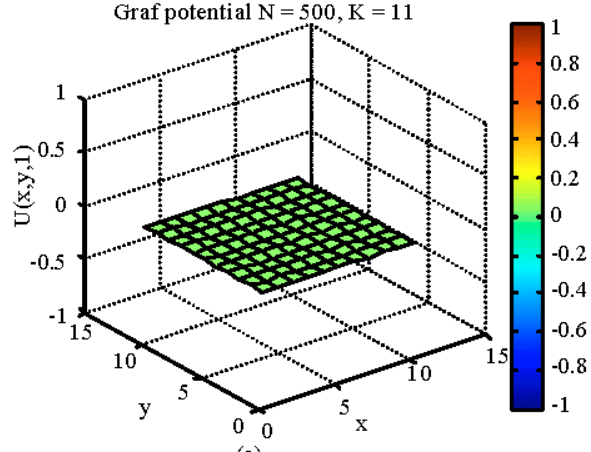

(a)

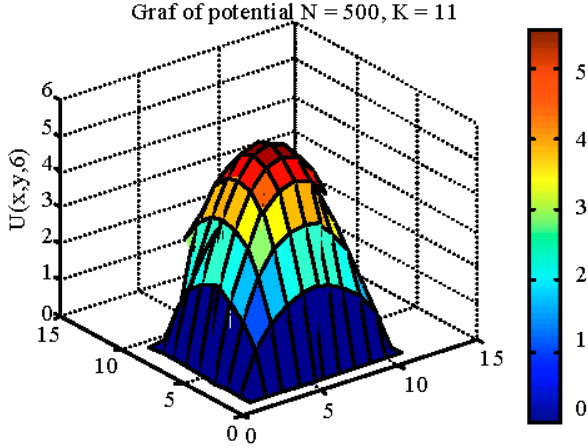

(b)

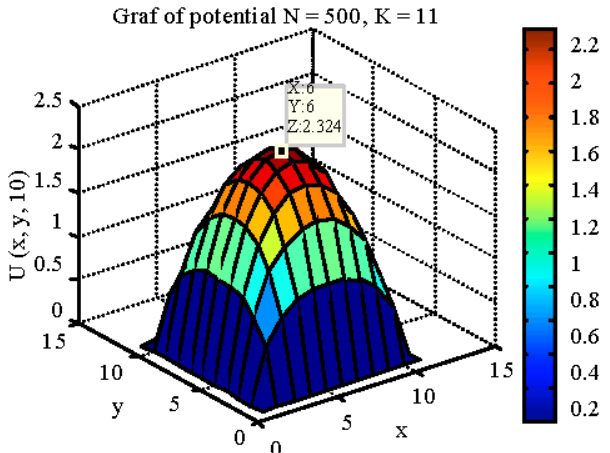

(c)

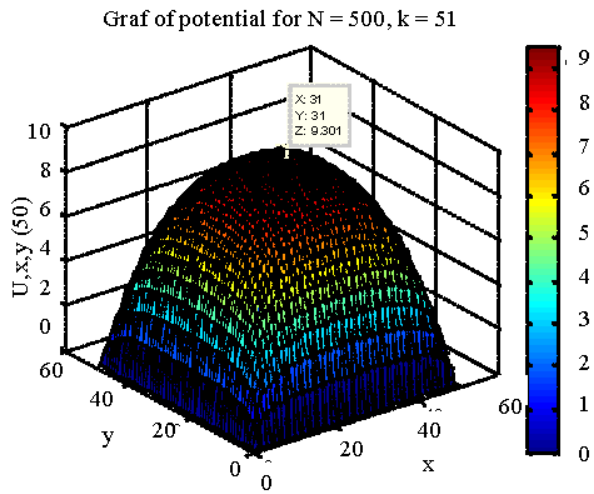

(d)

Fig. 4: Dependence of potencial: a) $\mathrm{z}=1, \mathrm{~b}) \mathrm{z}=6, \mathrm{c}) \mathrm{z}=10$, d) $\mathrm{z}=50$ 


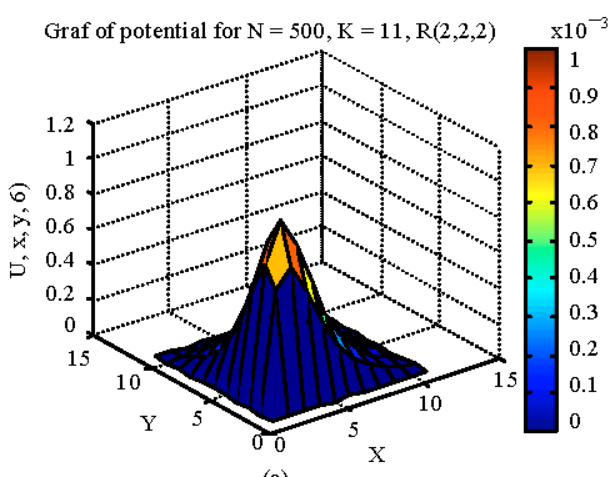

(a)

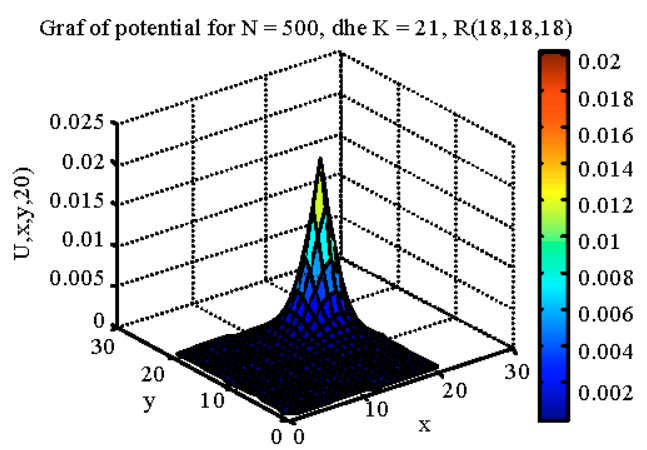

(b)

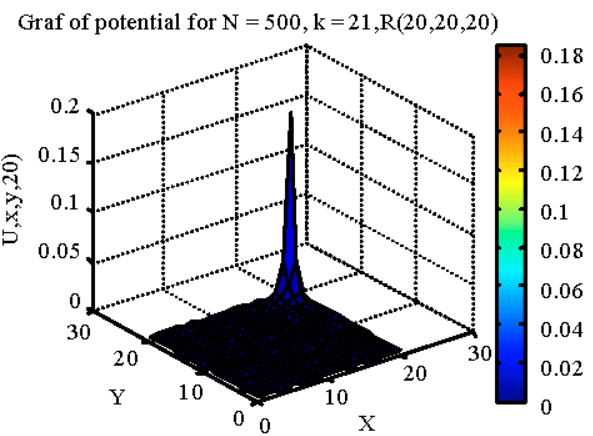

(c)

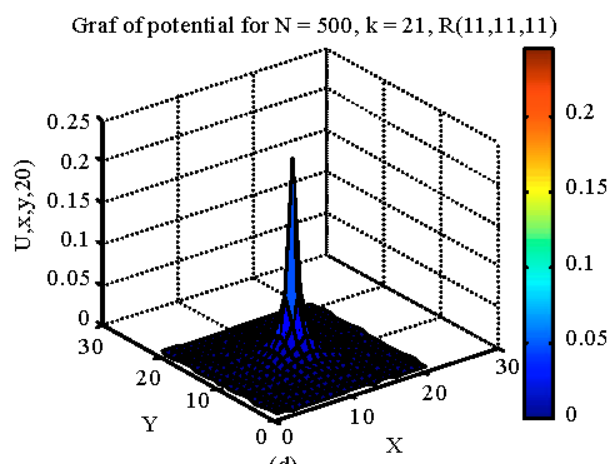

(d)

Fig. 5: Dependence of potential: a) $\mathrm{z}=6, \mathrm{~b}) \mathrm{z}=20$, point $(18,18), c) z=20$, point $(11,11), d) z=11$
- Inside the cube we suppose to have only one electric charge in any point and on other points inside the cube we don't have electric charges (Green's function) (Avdiaj, 2007).

Uniform distribution of electric charges: If the distribution of density charges in the cube is uniform, the form of potential inside the cube is displaced in the Fig. 4 and from those result we see that potential in the center is maximal and it going toward the edge this potential decreased due to of boundary condition. If we increased the number of grid points and the iterations the accuracy increased (Fig. 4).

Distribution of electric charges in certain points: If we have inside the cube only one electric charge we will obtain o-called Green function (Dirac delta function) (Fig. 5). Although again in this case the value of potential is higher near the electric charges.

\section{CONCLUSION}

In principle the partial differential equation of Poisson equation is solvable according to Finite Difference Method (FDM). The results that are obtained shown that maximum potential value appears at the center of cube, whereas potential values are reduced going toward cube edge. For the case of single electric charge inside the cube, a Green function (or a Dirac delta function) forms for the potential function appear. Accuracy of modeling increases with increasing the number of grid points and iterations.

\section{ACKNOWLEDGEMENT}

Presented research was partly financed by European Union, European Social Found. Operation implemented in the framework of Operational Program for Human Resources Development for the period 2007-2013, Priority axis1: Promoting entrepreneurships and adaptability, Main type of activity 1.1.: Experts and Researcher for competitive enterprises.

\section{REFERENCES}

Avdiaj, S., 2007. Solving of Electrostatics Problems. University of Tirana, Research seminar, Tirana, pp: 8-12.

Jackson, J.D., 1999. Classical Electrodynamics. 3rd Edn., John Wiley and Sons, New York, ISBN: 0-471- 30932X, pp: 24-70. 
Pang, T., 2006. An Introduction to Computational Physics. 2nd Edn., Cambridge University Press, New York, ISBN: 978-0-521-53276-1, pp: 197-225.

Roberts, A.J., 2001. Simple and fast multigrid solution of poissons equation using diagonally oriented grids. ANZIAM J., 43: E1-E36.
Sadiku, M.N.O., 2001. Numerical Techniques in Electromagnetics. 2nd Edn., CRC press, New York, ISBN: 0-8493-1395-3 pp: 134-231.

Smedingho, M.L., 2005. Solving the poisson equation with multigrid. http://cd-amr.fnal.gov/aas/poisson. pdf. 